\title{
Chemilche Umfchau
}

\author{
auf dem Gebiete der Fette, Oele, Wachse und Harze
}

(frther: Chemische Revue aber die Fett- und Harz-Industrie).

Zentral-Organ für die Industrien der Speise- u. der technischen Fette u. Oele, der Mineralöle u. Schmiermittel, des Stearins u. Glyzerins, der Kerzen, der Seifen, der Firnisse u. Lacke. Organ der wissenschaftlichen Zentralstelle für Oel- u. Fettforschung (e.V.) Berlin. Erscheint monalich zweimal. Deutsche Postzeitungsliste 1555.

Bezugspreis M. 48.- für das Jahr. Berechnung für das Ausland nach besonderem Tarif.

Alle die Schriftleitung betreffenden Mitteilungen an Dr. W. Fahrion, Feuerbach-Stuttgart, alle den Bezug und die Anzeigen betreffenden Zuschriften an den Verlag der Chemischen Umschau, Stuttgart, Postfach 40, erbeten. Nachdruck nur mit Genehmigung der Schriftleitung gestattet.

\begin{tabular}{lll}
\hline \hline XXVIII. Jahrgang. & Stuttgart, zweites Oktoberheft 1921. & Heft 20. \\
\hline
\end{tabular}

\section{Die Rückstände der Holzteerdestillation. \\ Von Prof. Dr. J. Marcusson und Dr. M. P. Picard.}

Die bei der trockenen Destillation des Holzes erhältlichen Teere finden zum Teil als solche Verwendung; so dient z. B. Nadelholzteer unmittelbar als Schiffsanstrich, zum Teeren von Tauen, Seilen usw. Vielfach wird aber auch eine erneute Destillation vorgenommen. Man gewinnt dabei aus Buchenholzteer Kreosot, aus Nadelholzteer das wertvolle Kienöl. Im Destillationsrückstande hinterbleiben grosse Mengen Pech, die als Asphaltersatz Verwendung finden. Ueber ihre Zusammensetzung war bis vor kurzem nur sehr wenig bekannt. Von Donath und Margosches ${ }^{1}$ ) war festgestellt, dass sich Holzteerpeche von allen ubrigen Pechen durch ihre Schwerlöslichkeit im Tetrachlorkohlenstoff unterscheiden. Diese Beobachtung kann sich aber nur auf sehr weit abgetriebene Proben beziehen, da sich dio Mehrzahl der von uns untersuchten weichen und mittelharten Holzteerpeche löste. Man wusste ferner, dass die Rückstände der Holzteerdestillation meist noch Phenole enthalten und demzufolge die Graefesche Diazobenzolchloridreaktion geben, dass ausserdem im Kienteerpech Harzsäuren vorkommen, welche sich durch beträchtliche Säurezahl und positiven Ausfall der Morawskischen Reaktion mit Essigsäureanhydrid und Schwefelsäure zu erkennen geben. Nachdem inzwischen von uns die Zusammensetzung der Holzteere aufgeklärt ist ${ }^{2}$ ), erschien es aussichtsvoll, auch in die Zusammensetzung der Holzteer-

1) Chem. Ind. 1904, 224.

2) Ztschr. t. angew. Chem. 34, 203 (1921). peche tiefer einzudringen. Die Holzteere hatten sich als ein wechselvolles Gemisch von unverseifbarem Oel, Oxysäuren und deren Anhydriden, Harzsäuren, Fettsäuren und Pbenolen erwiesen. Der chemische Aufbau der beiden wichtigsten Holzteere, Buchenholz- und Kienteer, geht aus der nachfolgenden Tabelle 1 hervor.

Tabelle 1.

Zusammensetzung von Buchenholz- und Kienteer.

\begin{tabular}{|c|c|c|c|c|c|c|c|}
\hline \multirow[b]{2}{*}{$\begin{array}{c}\begin{array}{c}\text { Art } \\
\text { des } \\
\text { Teeres }\end{array} \\
\end{array}$} & \multirow{2}{*}{$\begin{array}{l}\text { Unver- } \\
\text { self- } \\
\text { bares } \\
\% \\
\end{array}$} & \multirow{2}{*}{$\begin{array}{c}\text { Ashydride } \\
\text { van } \\
\text { oxy- } \\
\text { seuren } \\
\% \\
\end{array}$} & \multicolumn{2}{|c|}{ Oxystaturen } & \multirow{2}{*}{$\begin{array}{c}\text { Harz- } \\
\text { săuren } \\
\% \\
\end{array}$} & \multirow{2}{*}{$\begin{array}{c}\text { Fett- } \\
\text { săurea } \\
\% \\
\end{array}$} & \multirow[b]{2}{*}{$\begin{array}{c}\text { Phenole } \\
\% \\
\end{array}$} \\
\hline & & & $\begin{array}{c}\text { atherum- } \\
\text { Itosilen } \\
\% \\
\end{array}$ & $\begin{array}{c}\text { Sthes } \\
\text { Iaslen } \\
\% \\
\end{array}$ & & & \\
\hline $\begin{array}{l}\text { Bucher } \\
\text { hotztere }\end{array}$ & 18 & 9,5 & 38,3 & 19 & 7,7 & 3,2 & 9,3 \\
\hline $\begin{array}{l}\text { Klop- } \\
\text { teer }\end{array}$ & 53,5 & 0 & & 4 & 17 & 6 & 9,5 \\
\hline
\end{tabular}

Das im Anschluss an die Holzteeruntersuchung fur die Peche angewandte Prufungaverfahren war folgendes: Die Proben wurden mit uberschüssiger alkoholischer Kalilauge verseift, unlöslich bleibende Anteile (Teerharze) abgetrennt und ausgewaschen. Aus der alkoholischen Lösung wurde nach Spitz und Hönig das Unverseifbare ausgeschuttelt, dann wurde eingedampft, mit Wasser aufgenommen und angesäuert. Die ausfallenden Säuren waren zum Teil in Aether unlöslich, dunkelbraun gefärbt und bestanden aus $\mathrm{An}$ hydriden von Oxysäuren. Das Aetherlösliche konnte durch Benzin zorlegt werden. Benzinunlöslich verblieben Oxysäuren, die im Gegen- 
satz zu den ätherunlöslichen Anhydriden in Alkohol leicht löslich werden. Der benzinlösliche Rest enthieJt ein Gemisch von Phenolen, Fett- und Harzsäuren. Die Phenole wurden durch Behandeln mit heisser Sodalösung abgetrennt. In dieser lösten sich Fettund Harzsäuren als Salze auf, die unangegriffenen Phenole wurden mit Aether ausgezogen. Harz- und Fettsäuren wurden nach Twitchell getrennt.

Die Ergebnisse sind in Tabelle 2 zusammengestellt.

Tabelle 2.

Zusammensetzung von Holzteerriickständen.

\begin{tabular}{|c|c|c|c|c|c|c|c|c|}
\hline Materlal & $\begin{array}{l}\text { Kon- } \\
\text { sistonz }\end{array}$ & $\begin{array}{c}\text { Moy- } \\
\text { traic } \\
\text { Ieer- } \\
\text { barzo } \\
\% \\
\end{array}$ & $\begin{array}{c}\text { Un- } \\
\text { rerseif- } \\
\text { beres } \\
\% \\
\%\end{array}$ & $\begin{array}{c}\text { Phenolo } \\
\%\end{array}$ & $\begin{array}{l}\text { Oxy- } \\
\text { steren } \\
\text { uad fis- } \\
\text { hydrido } \\
\% \\
\%\end{array}$ & $\begin{array}{c}\text { Harz- } \\
\text { surea } \\
\% \\
\end{array}$ & $\begin{array}{c}\text { Fett- } \\
\text { stanen } \\
\% \\
\end{array}$ & $\begin{array}{c}\text { Mineral- } \\
\text { stoffe } \\
\% \\
\%\end{array}$ \\
\hline $\begin{array}{l}\text { Buabes- } \\
\text { holz- } \\
\text { tearpetel }\end{array}$ & $\begin{array}{l}\text { eprodt, } \\
\text { loleht } \\
\text { plyerl- } \\
\text { slorbar }\end{array}$ & 14 & 6 & 1,5 & 77 & 0 & 1,5 & - \\
\hline $\begin{array}{c}\text { Kientear- } \\
\text { poeh }\end{array}$ & 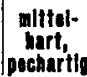 & 1,5 & 19,7 & 8 & 31,8 & 35,2 & 2,8 & 1 \\
\hline
\end{tabular}

Bemerkenswert ist der hohe Gehalt des Buchenholzteerpechs an Oxysäuren und deren
Anhydriden $(77 \%)$. Diese bedingen im wesentlichen den Pechcharakter. Im Kienteerpech sind dagegen neben Oxysäuren $(31,8 \%)$ erhebliche Mengen Harzsäuren (35\%) und fast $20 \%$ Unverself bares enthalten. Das Kienteerpech war in absol. Alkohol grösstenteils löslich, dagegen Ioste sich das Buchenholzteerpech weder in Alkohol, noch in Benzol oder Tetrachlorkohlenstoff. Es machte äusserlich den Eindruck, als ob bereits Verkokung eingetreten wäre. Erst die Behandlung mit heisser alkoholischer Lauge, bei der sich der grösste Teil löste, gab einen Einblick in den wahren Charakter des Materials.

Auf Grund vorstehender Ergebnisse lassen sich die Ruckstände der Holzteerdestillation, mögen sie nun aus Laubholz- oder Kienteer gewonnen sein, infolge ihres hohen Gehalts an Oxysäuren von allen anderen Kunstasphalten mit Sicherheit unterscheiden; zwar enthalten auch Fettpeche erhebliche Mengen von Oxysäuren, diese zeigen aber anderen Charakter, ausserdem fehlen den Fettdestillationsruckständen die fur Holzteerpeche charakteristischen Phenole.

\section{BERICHTE.}

\section{A. Theorie.}

Gljzeride des Gänsefettes. Brmer und Merten (vgl. Z. angew. Chem. S. 515) tanden im Gänsefett ausschliesslich Palmitin-, Stearin- und Oelsaure, Margarinsure (vgl. Klimont und Meyer, diese $Z$. 1915, 42) ist nicht vorhanden. Durch fraktionierte Lösung konnte $\mathrm{Palmitodistearin} \mathrm{in} \mathrm{geringer}$ Menge und ferner Dipalmitostearin isoliert werden. Die fllissigen Glyzeride bestehen vorwiegend aus Triolein und $P$ almitodiolein, daneben findet sich etwas Stearodiolein. Vgl. Amberger und B r o m ig, diese Z. H. 18 S. 233.

Die Fettsäuren des Erdnußöls (Jamieson, Baughman und Brauns, J. Amer. Chem. Soc. 43, 1372). Die Verf. untersuchten eingehend zwei selbst gepresste, nicht raffinierte amerikanische Erdnussöle, aus Samen, die in Karolina bzw. Virginia gewachsen waren (J.Z. nach Hanus 90,1 bzw. 94,8). Dio ungesättigten Fettsănren wurden in Form der Bromide, die gesattigten durch fraktionierte Vakuumdestillation der Methylester getrennt. Hypoga easäure, $\mathrm{C}_{16} \mathrm{H}_{30} \mathrm{O}_{3}$, konnte auch nicht in Spuren gefunden werden, trotzdem u. a. auch genau nach den Angaben von Schröder (Ann. Chem. 1867, 143, 22), owie von Gös mann und Scheren (Ann. Chem. 1855, 94, 230) und Caldwell und Gös s man n (Ann. Chem. 1856, 99, 305) gearbeitet wurde. Auch Linolensäure war nicht vorhanden, sondern nur $\mathrm{Oel-und} \mathrm{L}$ inols \&ure. Von gesüttigten Fettsauren wurde Myristinsäre nicht gefunden, Behen- säure erschien wahrscheinlich, doch konnte ihre $\mathrm{Ab}$ wesenheit nachgewiesen werden. Insgesamt wurden, nach Umrechnung der Fettsăuren auf die Giyzeride, folgende Gehalte gefunden (in \%)

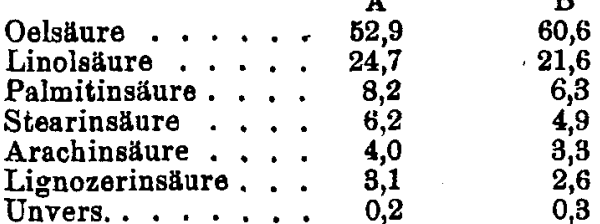

Diese Resultate weichen von denjenigen von Heiduschka und Felser (diese Z. 1920, 4) einigermassen ab.

0el des Sturmrogels (Mutton-bird, Aestrelata lessoni). Dieser Vogel ist auf den Inseln bei Tasmanien und Neuseeland heimisch. Wenn er gereizt wird, spritzt er sus seinen Nasenlöchern mit ziemlicher Kraft ein in seinem Magen enthaltenes Oel, das ausserdem auch als Nahrung für die jungen Vögel dienen soll. Dieses Oel wurde von C. L. Carter (J. Soc. Chem. Ind. 40, 220) paher untersucht und als dem Spermazeto sehr ahnlich befunden. Es enthalt $38,4 \% Z$ et $y !$. alkohol, Smp. $31-31,5^{\circ}$, Smp. des Azetats $18,5^{\circ}$. Von den Fettsäuren sind nur $3,3 \%$ fest, die flüssigen bestehen hauptsächlich aus 0 e 1 a ä $\mathrm{r} e$, müsseu aber, wie aus der J. Z. 178 und der positiven Hexabromidreaktion hervorgeht, auch stärker ungesaltigte Fott sluren entbalten. Durch Pankreatin wird das Oe 